\title{
A Novel Implant Electrode Design Safe in the RF Field of MRI Scanners
}

\author{
Steven McCabe and Jonathan Scott, Senior Member, IEEE
}

\begin{abstract}
Patients with medical implants are often unable to receive Magnetic Resonance Imaging (MRI) diagnostic treatment because the conductive leads can concentrate the RF excitation field and generate dangerous heating of nervous tissue. We propose a simple low-cost solution that minimizes RF heating through the addition of one or more mutually-coupled filars to the lead without significant increase in lead diameter. Simulations and measurements at $128 \mathrm{MHz}$ are presented to verify the effect in 3-Tesla MRI machines.
\end{abstract}

Keywords-Biomedical electrodes, medical diagnostic imaging, electrical stimulation, electromagnetic modeling, specific absorption rate, heating, safety.

\section{INTRODUCTION}

$\mathbf{T}$ HE RF field generated by the transmit coils in a Magnetic Resonance Imaging (MRI) machine can lead to hazardous heating of the patient [1]. Machines with 3 Tesla magnets are capable of delivering $128 \mathrm{MHz}$ RF pulses of several tens of $\mathrm{kW}$ [2]. Normally the risk of heating is mitigated through active monitoring and control of the Specific Absorption Rate (SAR), defined as the rate of RF power absorption per object mass over a set exposure time [3]. The object mass is specified as either the whole-body, whole-head, or in situations where localized hot spots can occur, $10 \mathrm{~g}$.

This safety measure is greatly inadequate for patients with medical implants, particularly those comprising long electrode leads as found in Deep Brain Stimulation (DBS) and Spinal Cord Stimulation (SCS) implants. Fig. 1 shows the distal end of a typical lead for SCS. Such leads can range up to several hundred millimeters in length, or $0.5-2$ wavelengths in vivo [4]. Under the RF field from MRI, the lead conductors can resonate as antennas and transfer RF energy into highly concentrated regions of tissue near the distal electrodes [5], [6]. The absorbed energy can generate significant heating and cause damage to the nervous tissue. Simulations reported in [7] reveal distal heating from MRI can concentrate into tissue mass of less than $1 \mathrm{~g}$.

Radiographers from around the world routinely consult the online database in [1] to check if an implant has been tested and rated "safe" or "conditionally safe" before scanning the patient. Despite a spate of patent filings over the last decade,

\footnotetext{
Manuscript received July 01, 2016; revised December 22, 2016; accepted December 28, 2016.

The authors are with the Department of Engineering, The University of Waikato, Hamilton 3240, New Zealand (email: stowmc@gmail.com; scottj@waikato.ac.nz).

Color versions of one or more of the figures in this paper are available online at http://ieeexplore.ieee.org.

Digital Object Identifier
}

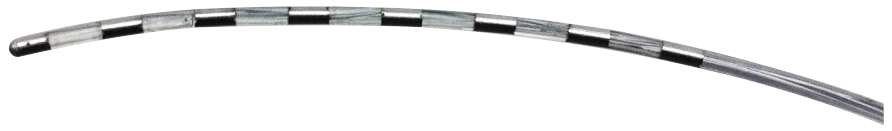

Fig. 1. The distal end of an SCS lead with eight platinum electrodes. Each electrode is $3 \mathrm{~mm}$ in length, $1.3 \mathrm{~mm}$ in diameter, and spaced apart by $4 \mathrm{~mm}$ of insulation. Insulated platinum filars extend through the length of the lead to each individual electrode.

see [8] through [15], only one product is available that has been approved for use in MRI machines. The SCS system, developed by Medtronic is rated conditionally safe. It is limited to $1.5 \mathrm{~T}$ machines and requires a restrictive scanning protocol. Currently, there are no DBS products available that offer any MRI compatibility.

This manuscript introduces a new technique to improve the RF safety of implants in MRI machines and is an expansion of [16]. The technique, termed "decoy", requires as little as a single extra filar to be incorporated externally along the implant lead. It works by means of mutual coupling to an added filar of selected length and with thin insulation [6], [7], [16], [17]. When combined with resistive materials [6], [7] and surface treatments [18], it is expected to achieve superior MRI-safe performance with minimal scanning restrictions. The approach is explored in detail in section III. In section IV we expand upon the single electrode work from [16] to include measurements on leads with multiple electrodes. We explore the impact of increasing the number of electrodes and demonstrate the effectiveness of adding decoy conductors to the lead.

\section{INSULATED WIRE}

Complex multi-electrode structures are generally modelled as simple single-core wire structures [5]-[7], [19]-[21] to avoid excessively long simulation times and impractical memory and CPU resource requirements. Most experiments are also limited to these simpler structures to help minimize the running time and costs associated with MRI machines. Fig. 2 compares the distal heating predicted by simulation with that measured in the commercial $3 \mathrm{~T}$ MRI machine in Fig. 3, for a whole-body SAR of $1 \mathrm{~W} / \mathrm{kg}$. For simulation details refer to section III. The International Commission on Non-Ionizing Radiation Protection (ICNIRP) [22] recommends a threshold of $1-2{ }^{\circ} \mathrm{C}$ for tissue heating, but this level is exceeded more than ten times in the worst case. Enclosing the lead within a cylindrical metallic shield results in a significant reduction of heating as is shown in the same figure, and is the key mechanism that achieves MRI-compatibility in the commercial leads by Medtronic. 


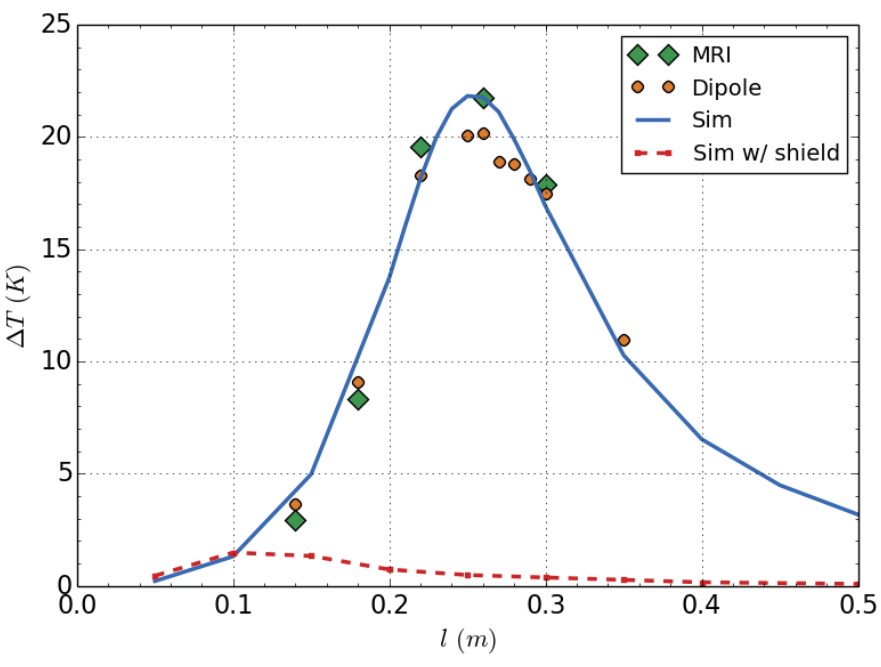

Fig. 2. Simulation and measurement of the temperature rise $\Delta T$ near the distal electrode of an insulated $800 \mu \mathrm{m}$ dia. copper wire after 5 minutes of scanning in a 3T MRI machine [23]. Plastic insulation $350 \mu \mathrm{m}$ in thickness covers all but the $6 \mathrm{~mm}$ electrode at one end. Simulation of the same lead but with a $100 \mu \mathrm{m}$ thick platinum shield, is shown by the dashed line.

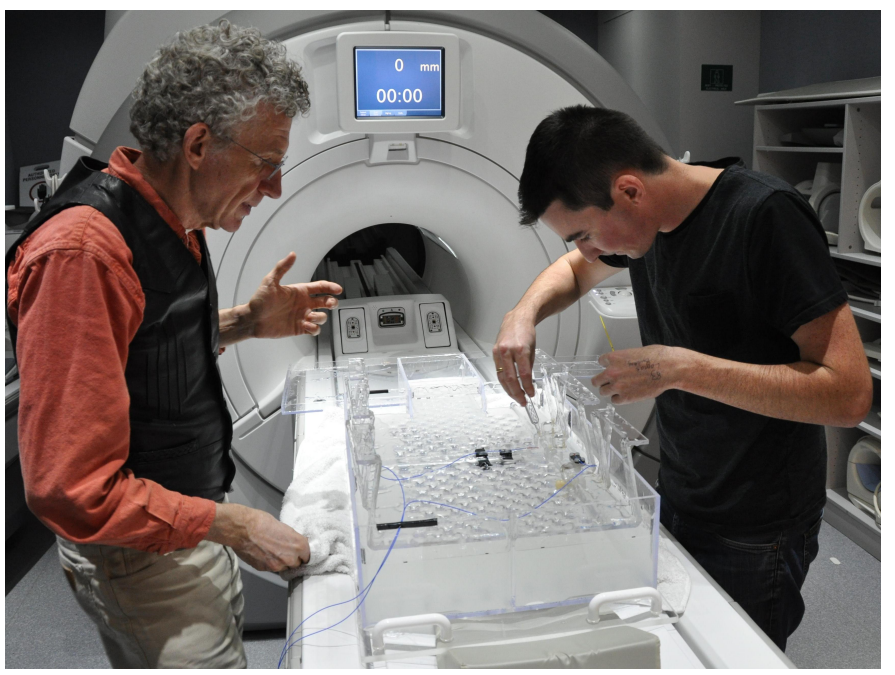

Fig. 3. Operators position an implant lead inside of a gelled saline phantom on the bed of a $3 \mathrm{~T}$ MRI machine. A GaAs-based fiber-optic temperature probe provides measurement of the distal electrode heating.

Recent work in [4] and [23] has demonstrated the use of dipole radiators in place of an MRI machine to predict the level of heating induced by implant leads. Included in Fig. 2 are measurements made using $128 \mathrm{MHz}$ dipole radiators and a $30 \mathrm{~W} \mathrm{CW}$ power amplifier. The results are consistent with expectations and provide a handy tool for rapid evaluation of design changes.

\section{Insulated Wire With Mutually-COUPLED DECOY}

The level of distal heating from an implant is proportional to the magnitude of current induced in the lead filars. Works by [6], [7] and [24] have shown that an increase in filar resistance to several hundred per meter can significantly reduce RF currents and heating. Furthermore, the incorporation of series inductance [20], [24], [25], metal sheaths [14], [15], and shunt capacitance [6], [7] can also help impede or divert current flow. Seemingly lacking from the literature, are the effects mutual coupling between adjacent lead filars can have on the induction of RF currents from MRI.

Mutual coupling is an inherent mechanism of antennas, with influence only in the near-field. Yagi-Uda antennas utilize the effect to couple directors and reflectors with a driven dipole to achieve high directionality [26]. Similarly, implant leads can exhibit antenna-like behavior. An electrode is effectively an antenna embedded in matter, a subject that has been dealt with in detail by King [27].

When an additional and preferably bare conductive wire is attached to the exterior of the insulated wire from section II, the pair will become mutually coupled at the MRI RF frequency. This ancillary filar is hereinafter referred to as a decoy. The intimate contact between the decoy filar and surrounding tissue provides a damping effect, an idea that has been reported in the literature [6], [7], and is mirrored by the insulated wire through mutual coupling [17]. Induced RF currents in the insulated wire are therefore smaller, resulting in abated power dissipation and joule heating at the electrode-to-tissue interface.

\section{A. Simulation}

Simulations were performed in COMSOL Multiphysics 4.4 using a phantom model set according to ASTM F218211a [28]. Excitation was provided by a 16 rung $128 \mathrm{MHz}$ birdcage as in [6]. The implant lead was orientated $5.5 \mathrm{~cm}$ from the phantom's left side and centered longitudinally and vertically within the torso. With the exception of the lead dimensions, the simulation parameters correspond to those previously reported in [6]. Simulation predicts that the current induced in a single conductor mostly insulated from the surrounding medium will be significantly reduced if the first filar is coupled to a second, uninsulated filar. The current is also reduced if the second filar is only thinly insulated, and reduced still further if two mutually-coupled uninsulated filars are deployed. See Fig. 4.

Fig. 5(a) plots predictions of distal heating along leads of various lengths in the presence of a single uninsulated decoy of specified proportionate length. It is apparent that an uninsulated filar with length $0.6-0.7$ times the length of the insulated filar leads to minimal heating over the range. The resonant length is not only dependent on the thickness and permittivity of the insulating material [6], [7], but also varies with decoy length as is shown in the same figure.

Heating problems are almost entirely resolved when leads have two uninsulated decoys of length between $0.7-0.9$ times the length of the main filar. See Fig. 5(b). Although the decoys themselves experience some heating along their length, simulation predicts it will be less than the heating of the distal stimulus electrode, except for short leads with $0.9 l$ decoys. This aberration is a result of the combined heating that occurs 


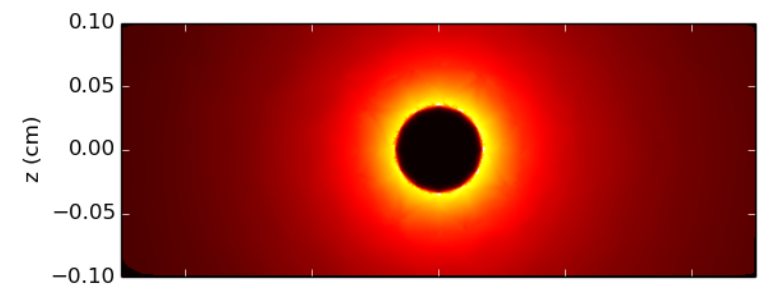

(a)

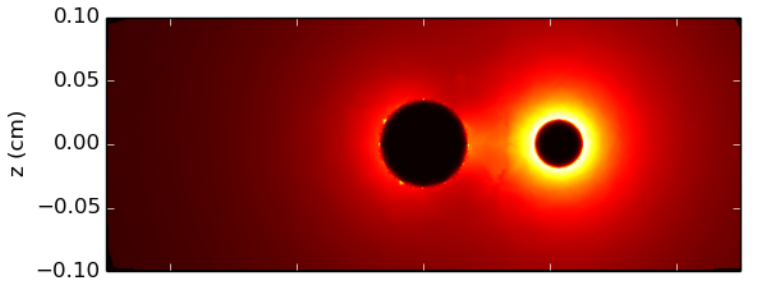

(b)

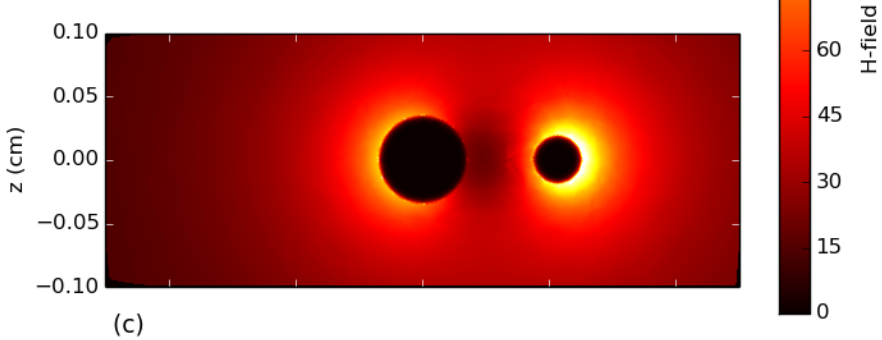

(c)

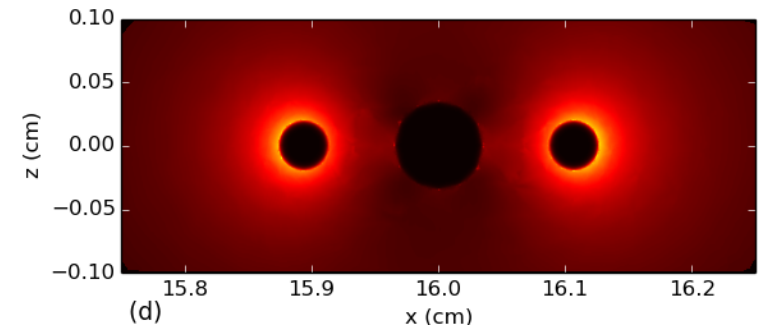

Fig. 4. Mid-point cross section displaying the simulated magnitude of the $\mathrm{H}$-field surrounding an (a) insulated wire (b) insulated wire with single bare $0.6 l$ decoy (rightmost conductor) (c) insulated wire with single $0.6 l$ decoy coated with $21 \mu \mathrm{m}$ thick insulation (rightmost conductor) (d) insulated wire with pair of bare $0.9 l$ decoys (outermost conductors). The combined length, $l$, of the insulated wire and $6 \mathrm{~mm}$ bared end is $25 \mathrm{~cm}$.

when the proximity between the decoy and electrode is close and will be highlighted in Figs. 9(b) and 10(b).

\section{B. Measurement}

The experimental set-up from [4] and [23] was replicated and is shown in Fig. 6. Dipole radiators are positioned alongside the lead under test and driven by a $128 \mathrm{MHz} \mathrm{CW}$ through a $30 \mathrm{~W}$ RF power amplifier. An Optocon FOTEMP thermometer monitors the temperature of the distal electrode using a GaAsbased fiber-optic probe with $0.1^{\circ} \mathrm{C}$ resolution. The experiment is calibrated for a whole-body SAR of $1 \mathrm{~W} / \mathrm{kg}$.

Referring to Fig. 7, example leads with diameters and insulation thickness as previously reported in [4], [7], [23] were fitted with bare decoy filars. This arrangement will be used to compare simulation with measurement in both an MRI
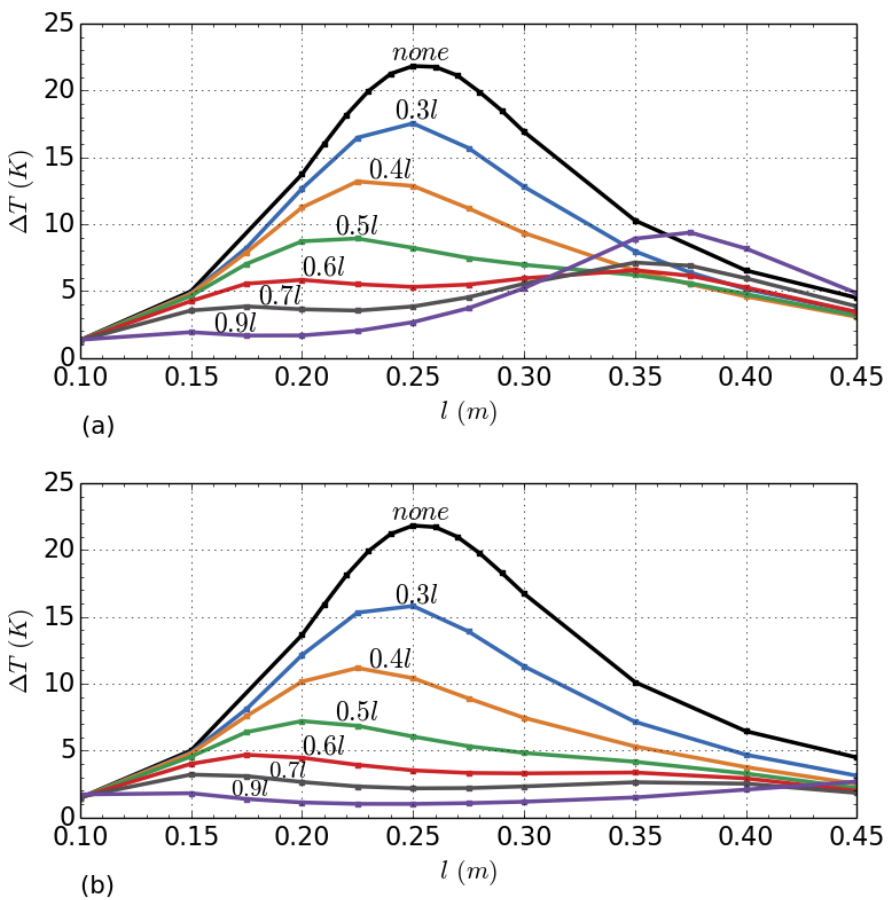

Fig. 5. Simulated distal heating $\left(t_{f}=5 \mathrm{~min}, \mathrm{SAR}_{w b}=1 \mathrm{~W} / \mathrm{kg}\right)$ as a function of wire length, for a range of leads each with (a) a single decoy (b) two decoys. Leads differ by a fixed decoy-to-lead length ratio in the range of $0.3-0.9 l$. The heating of an ordinary insulated wire without decoys is labeled with "none".

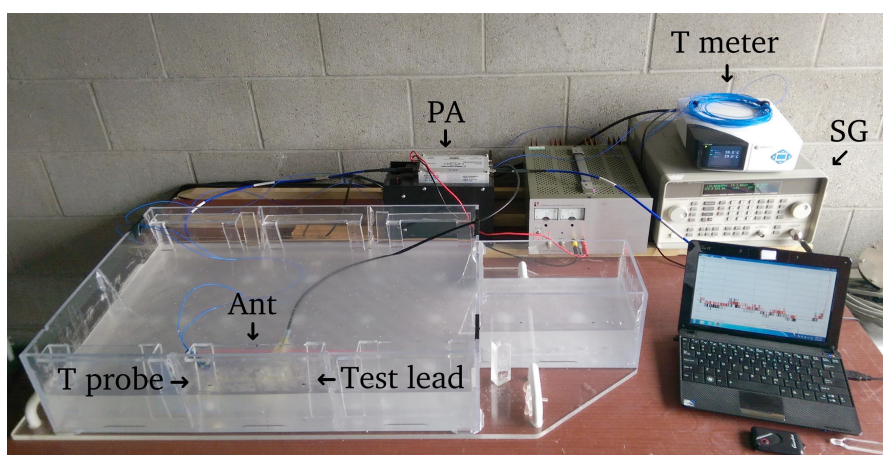

Fig. 6. Calibrated experimental set-up to measure RF heating in the lab.

machine and in our lab setup. Alignment of the temperature probe to the bared end of the lead under test is shown in Fig. 8. This type of probe is standard for monitoring implant heating in MRI machines, and is not expected to have a significant impact on the RF field and level of heating.

Measurements of peak distal heating were made on wires with a single $0.6 l$ decoy and a $0.9 l$ decoy. Results appear in Fig. 9. In the case of the shorter decoy, a temperature rise of less than $6^{\circ} \mathrm{C}$ over the entire lead is observed. The fractionallylonger decoy produces a smaller temperature rise-just $2^{\circ} \mathrm{C}$ when the lead length is short. The simulated predictions taken from above overlay measured data to permit visual comparison. Agreement is excellent. 


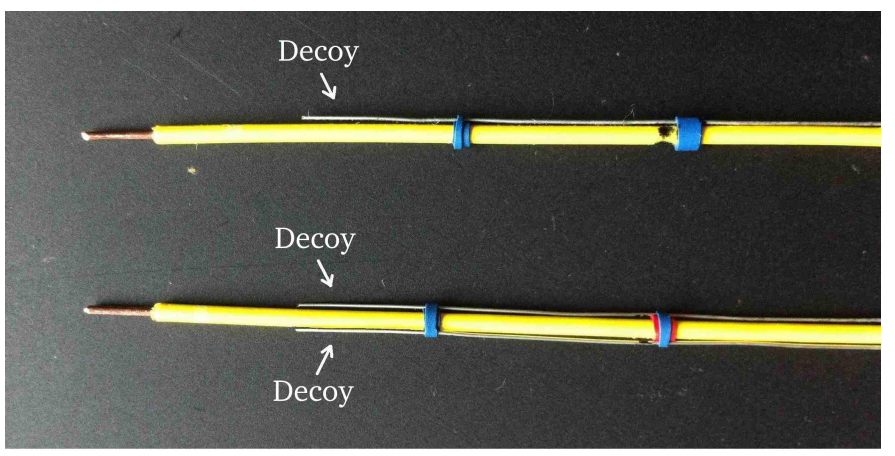

Fig. 7. Test leads comprising a single decoy (top) and pair of decoys (bottom) were constructed from $800 \mu \mathrm{m}$ dia. copper wire with plastic insulation $350 \mu \mathrm{m}$ in thickness covering all but $6 \mathrm{~mm}$ from one end (the electrode). Bare $400 \mu \mathrm{m}$ dia. copper decoy(s) are adhered to the exterior of the lead insulation.

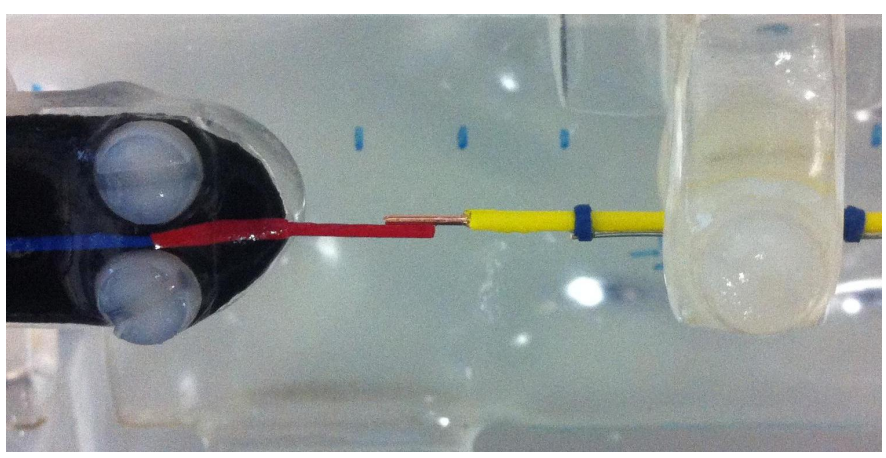

Fig. 8. GaAs-based fiber-optic temperature probe aligned with the bared end of the test lead.

As stated previously, there is a special case of when the distal electrode is not the source of highest heating. When the length of the $0.9 l$ lead is short the decoy itself generates slightly higher heating than the distal electrode. This is indicated in the figure by the result labelled "corrected" as shown in Fig. 9(b). A small elevation occurs for lead lengths up to $22.5 \mathrm{~cm}$. Measurement of the decoy was not performed due to difficulty in measuring the temperature of such a large volume.

A second decoy of identical length leads to an even greater reduction in distal heating. Referring to Fig. 10, it can be seen that the effectiveness is similar to the shielded, insulated wire in Fig. 2. A rise of just $0.5^{\circ} \mathrm{C}$ is measured when the lead is $30 \mathrm{~cm}$ long with $0.9 l$ decoys. This represents only $3 \%$ of the heating observed in the case of a plain implant lead as presented in Fig. 2. Decoy temperatures slightly warmer than the distal electrode are again predicted for the $0.9 l$ lead, for lead lengths up to $22.5 \mathrm{~cm}$.

\section{Multi-Electrode Implant Lead}

We now expand our analysis to complex lead structures that more closely represent commercial implant electrode leads. Implant leads for neuro-stimulation like the type shown in Fig. 1 typically employ $4-8$ electrodes for stimulus. Literature concerning the RF effects of such leads is limited to fixed lengths [25], [29]-[31]. With recent development of the lab test
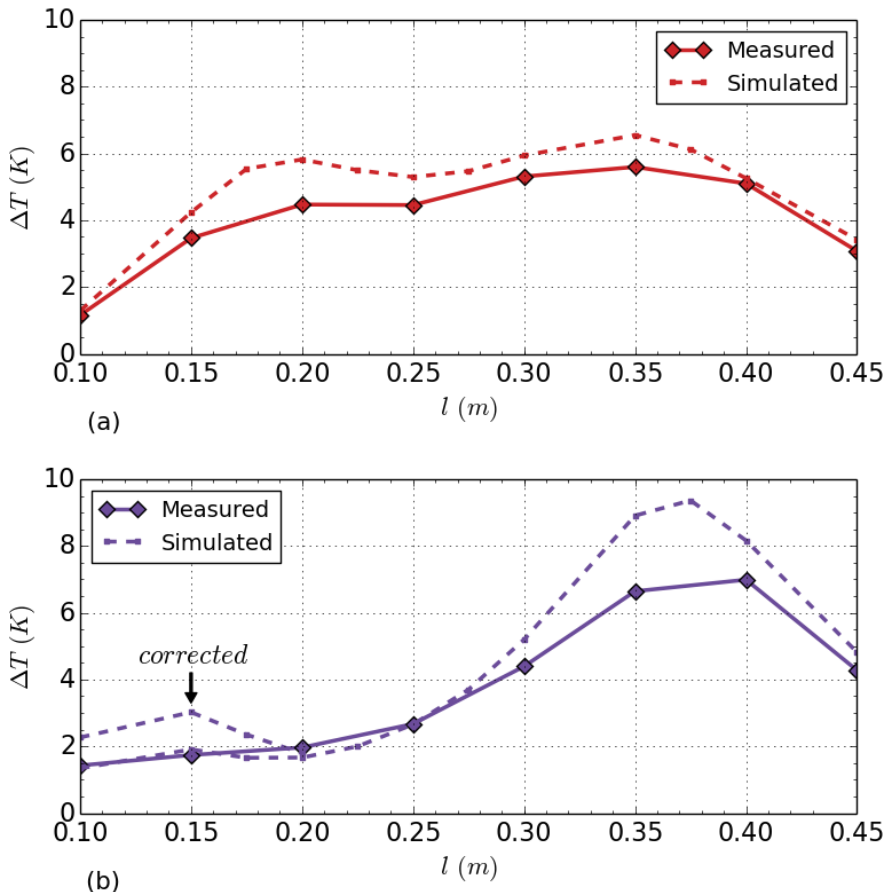

Fig. 9. Measured distal heating ( $t_{f}=5 \mathrm{~min}, \mathrm{SAR}_{w b}=1 \mathrm{~W} / \mathrm{kg}$ ) of single decoy-enhanced test leads, with decoy-to-lead length ratios of (a) $0.6 l$ and (b) $0.9 l$.
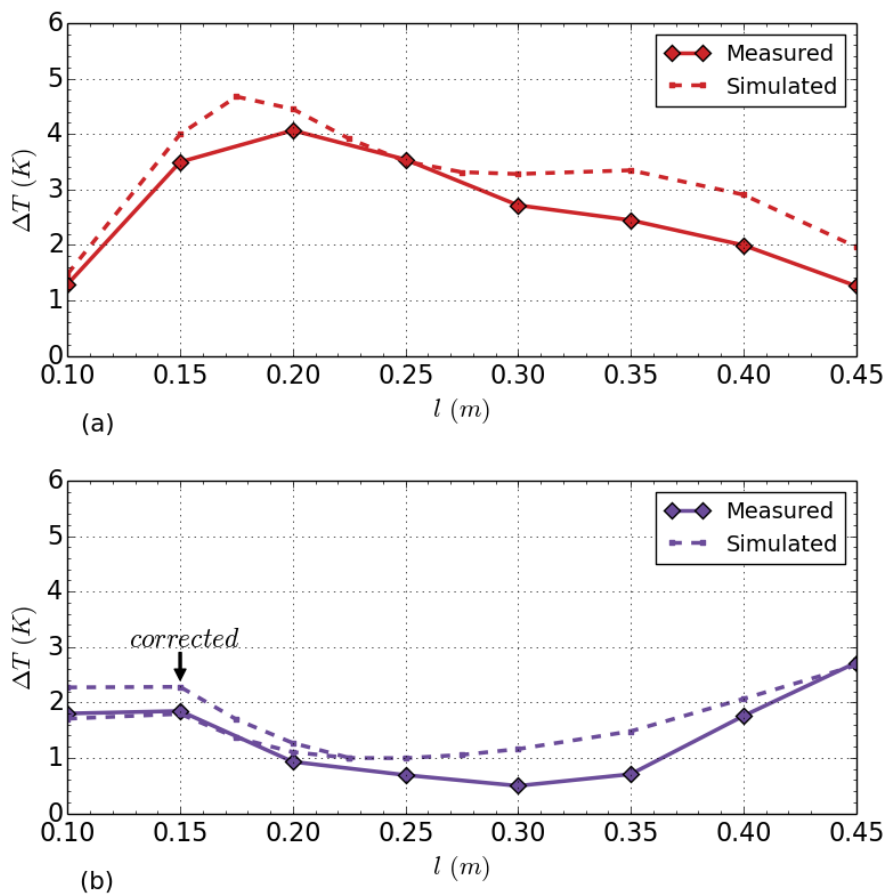

Fig. 10. Measured distal heating $\left(t_{f}=5 \mathrm{~min}, \mathrm{SAR}_{w b}=1 \mathrm{~W} / \mathrm{kg}\right)$ of dual decoy-enhanced test leads, with decoy-to-lead length ratios of (a) $0.6 l$ and (b) $0.9 l$. 
TABLE I. DETAILS OF THE DIFFERENT LEAD TYPES

\begin{tabular}{|c|c|c|}
\hline No. of electrodes & Cross section & Filar dia. $(\mathrm{mm})$ \\
\hline 1 & & 0.80 \\
\hline 4 & & 0.45 \\
\hline 8 & & 0.30 \\
\hline 8 & & $0.30 \mathrm{w} / 0.40 \mathrm{decoys}$ \\
\hline
\end{tabular}

method, extensive testing of multi-electrode leads has become feasible. We will now explore the effect of increasing the electrode count and further verify the decoy approach.

\section{A. Lead Types}

Single, four, and eight electrode versions were constructed with dimensions according to the SCS lead in Fig. 1, but with slightly larger outer diameters of $1.6 \mathrm{~mm}$. Straight lengths of enamel coated copper wire were soldered to each cylindrical electrode. The bundle of filars was sealed within a plastic sheath $350 \mu \mathrm{m}$ in thickness. Epoxy resin was applied to seal the opposing end of the sheath.

To avoid any influence on heating from varied insulation thickness or lead diameter, different diameters for the filars were chosen such that the sheath thickness and outside diameter for each lead type was equivalent. Design details for each lead type are summarized in Table I. A second eight electrode lead was also constructed but with the addition of two $0.9 l$ $400 \mu \mathrm{m}$ diameter decoys adhered on either side of the lead.

\section{B. Measurement}

Fig. 11 depicts the GaAs-based temperature probes aligned to the distal electrodes of the $45 \mathrm{~cm}$ eight electrode lead in the lab. Lead length is measured from the mid-point between the electrode set (between electrodes 4 and 5 for the 8-electrode version), to the opposite end of the lead. With the thermometry system supporting only two probes, the heating of just two electrodes could be measured at any one time. Following each complete set of measurements, the end of the lead was cut to form a new length, epoxy resin applied, and the measurement procedure repeated.

The measured heating for the lead with the single electrode is shown in Fig. 12(a). The highest temperature occurs when

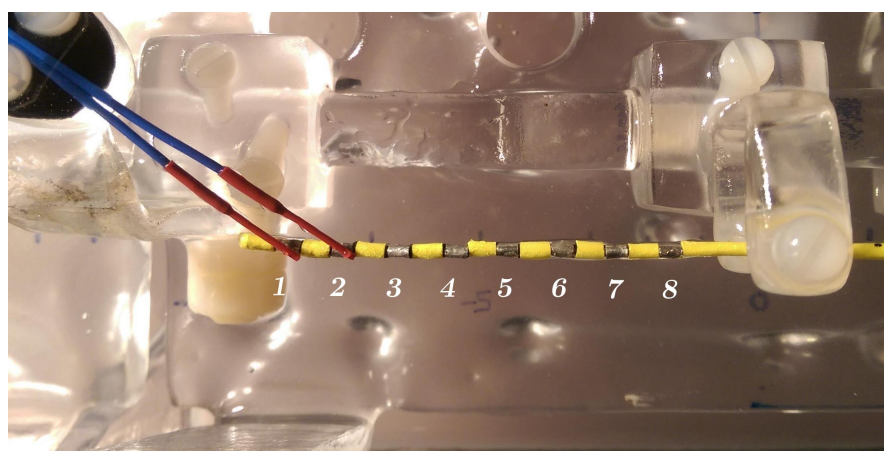

Fig. 11. Temperature probes aligned and in direct contact with the first two electrodes of the implant lead. Assigned numbering starts from " 1 " at the most distal electrode.

the length is $25 \mathrm{~cm}$, consistent with Fig. 2. Figs. 12(b) and 12(c), show measurements for the four and eight electrode versions, respectively. It is apparent from the figures that increased electrode counts lead to lower peak heating, where the most distal electrodes account for the highest heating. Fig. 12(d) shows the safety gained from adding two $0.9 l$ decoys to the eight electrode lead. Minimal heating occurs when the lead is short but is still within ICNIRP limits. The results are compiled into Fig. 13 to show the differences between maximum heating observed for each lead type.

\section{DISCUSSION}

Implant leads with higher electrode counts tend to generate less RF heating. This can be attributed to mutual coupling effects between individual filars and the apparent increase in electrode surface area gained from closely spaced electrodes. Electrodes other than the most distal ones produce the least amount of heating and can effectively be omitted from tests.

To virtually eliminate all heating and achieve MRI safety, leads can be fitted with external decoys to help dampen the induced currents generated in the lead filars. The modification is simple and cost-effective, and has no impact on the normal operation of the neuro-stimulation device. Extensive testing following [32] with more complex human phantoms is recommended including an assessment on decoy performance versus lead position/configuration.

Further improvement to the safety margin may be possible by increasing the ac resistance and surface area of the lead filars and decoys through the use of resistive materials [6], [7] and surface roughening techniques [18].

\section{CONCLUSION}

We presented a novel technique to ameliorate the potentially-hazardous heating of implanted conductors that can occur in MRI scanners. Simulation of local heating around the distal end of a filar agree with measurements made in a torso phantom. This mechanism of mutual coupling to a damped filar or "decoy" can be deployed in conjunction with other, previously-reported techniques. It is expected the approach will lead to MRI-safe leads for use with spinal cord and deep brain implantable stimulators. 

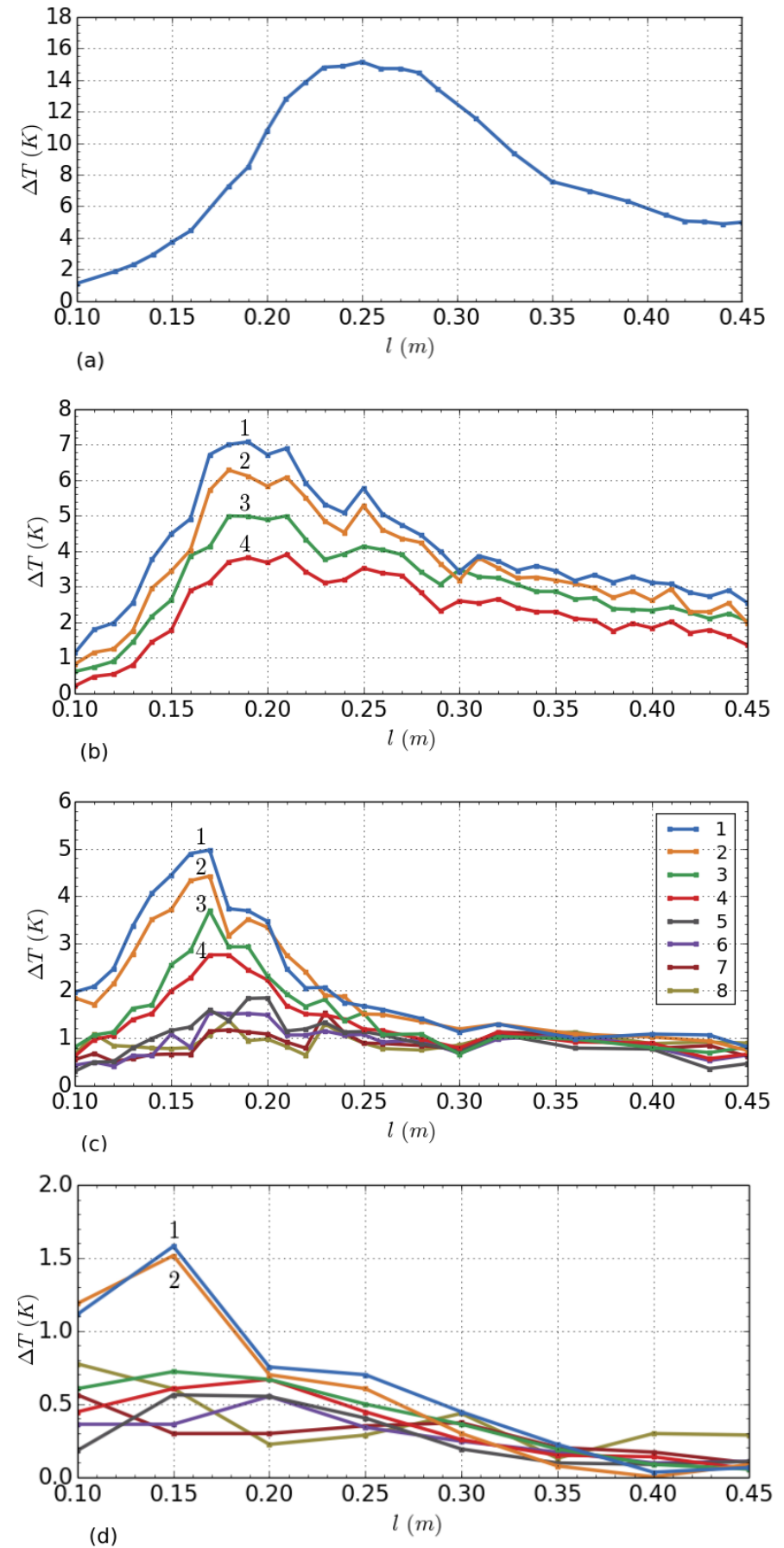

Fig. 12. Measured distal heating $\left(t_{f}=5 \mathrm{~min}, \mathrm{SAR}_{w b}=1 \mathrm{~W} / \mathrm{kg}\right)$ of an implant lead with (a) a single electrode (b) four electrodes (c) eight electrodes (d) eight electrodes with two $0.9 l$ decoys.

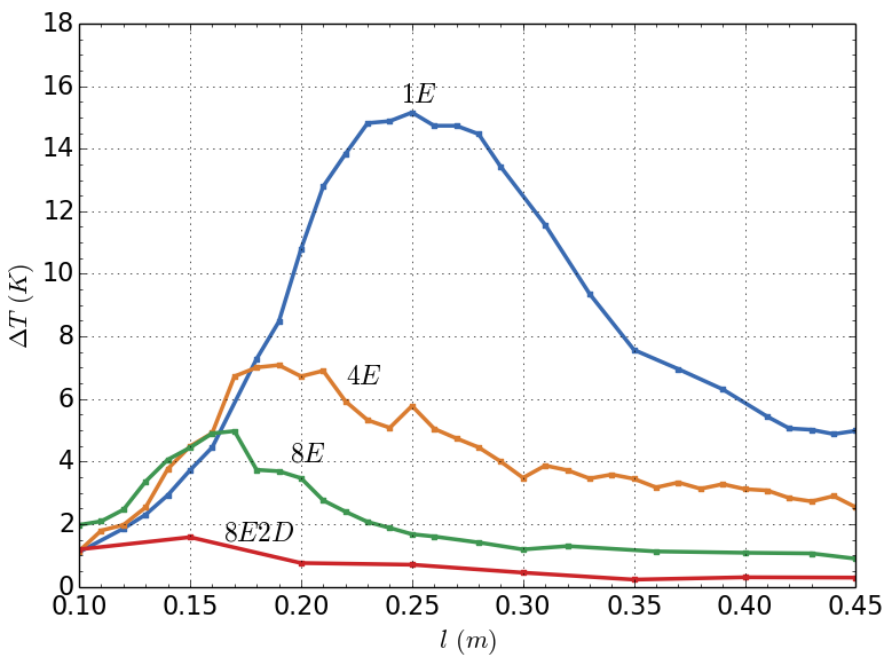

Fig. 13. Highest heating for the four and eight electrode versions compared with the single electrode lead.

\section{ACKNOWLEDGEMENT}

The authors would like to acknowledge Saluda Medical for their valuable support and Stephen Butler from Midland MRI for assisting with MRI experiments.

\section{REFERENCES}

[1] "MRIsafety.com, your information resource for MRI safety, bioeffects, \& patient management," http://www.mrisafety.com/ retrieved Jan. 2017.

[2] R. Caverly, G. Breed, W. Cantrell, M. Eron, J. Garcia, N. Kondrath, D. Myer, M. Ruiz, and J. Walker, "Advancements at the lower end: Advances in HF, VHF, and UHF systems and technology," IEEE Microw. Mag., vol. 16, no. 1, pp. 28-49, Feb. 2015.

[3] R. H. Caverly, "MRI fundamentals," IEEE Microw. Mag., vol. 16, no. 6, pp. 20-33, Jul. 2015.

[4] S. McCabe and J. Scott, "Measurement of implant electrode leads using time-domain reflectometry to predict the resonant length for MRI heating," Proceedings of the 22nd Electronics New Zealand Conference, Wellington, 17-18 Nov. 2016, pp. 90-94.

[5] J. A. Nyenhuis, P. Sung-Min, R. Kamondetdacha, A. Amjad, F. G. Shellock, and A. R. Rezai, "MRI and implanted medical devices: basic interactions with an emphasis on heating," IEEE Trans. Device and Mater. Rel., vol. 5, no. 3, pp. 467-480, Sep. 2005.

[6] S. McCabe and J. Scott, "Cause and amelioration of MRI-induced heating through medical implant lead wires," Proceedings of the 21st Electronics New Zealand Conference, Hamilton, 20-21 Nov. 2014, pp. $34-40$.

[7] S. McCabe and J. Scott, "Electromagnetic techniques to minimize the risk of hazardous local heating around medical implant electrodes during MRI scanning," European Microwave Conference, Paris, 7-10 Sep. 2015, pp. 702-705.

[8] C. D. Wahlstrand and T. B. Hoegh, "Electrode for use in an MRI-safe implantable medical device," European patent number EP 1742701 B1, Aug, 2010.

[9] C. D. Wahlstrand, G. A. Hrdlicka, R. M. Skime, P. Przybszewski, and T. E. Cross, "Energy shunt for producing an MRI-safe implantable medical device," International patent number WO 2005/030322 Al, Apr, 2005. 
[10] J. M. Olsen, S. L. Bolea, G. A. Hrdlicka, C. D. Wahlstrand, and T. B. Hoegh, "Lead electrode for use in an MRI-safe implantable medical device," International patent number WO 2006/093685 A1, Sep, 2006.

[11] B. A. Tranchina, "Method for fabricating a stimulation lead to reduce MRI heating," US patent number 8,601,672 B2, Dec, 2013.

[12] G. Bonmassar and E. Eskandar, "MRI compatible leads for a deep brain stimulation system," International patent number WO2014055737 Al, Apr, 2014.

[13] C. D. Wahlstrand, R. M. Skime, G. A. Hrdlicka, J. M. Olsen, and S. L. Bolea, "MRI-safe implantable medical device," US patent number 8,620,453 B2, Dec, 2013.

[14] T. B. Hoegh, S. L. Bolea, C. D. Wahlstrand, G. A. Hrdlicka, and J. M. Olsen, "Lead electrode for use in an MRI-safe implantable medical device," European patent number EP 1740260 B1, Aug, 2010.

[15] T. B. Hoegh, S. L. Bolea, C. D. Wahlstrand, G. A. Hrdlicka, and J. M. Olsen, "Lead electrode for use in an MRI-safe implantable medical device," US patent number US 8,364,286 B2, Jan, 2013.

[16] S. McCabe and J. Scott, "New MRI-safe implant electrode design," International Microwave Symposium, San Francisco, 22-27 May. 2016, pp. $1-3$.

[17] S. O. McCabe and J. B. Scott, "Implant conductor assembly with improved radio frequency properties," New Zealand provisional patent 714212, Filed on Nov. 16, 2015.

[18] S. O. McCabe and J. B. Scott, "A surgical implant conductor with increased radio frequency alternating current resistance," International patent number WO 2016/195513 A1, Dec, 2016.

[19] C. D. Smith, A. V. Kildshev, J. A. Nyenhuis, and K. S. Foster, "Interactions of magnetic resonance imaging radio frequency magnetic fields with elongated medical implants," J. Appl. Phys., vol. 87, no. 9, pp. 6188-6190, May. 2000.

[20] E. Cabot, T. Lloyd, A. Christ, W. Kainz, M. Douglas, G. Stenzel, S. Wedan, and N. Kuster, "Evaluation of the RF heating of a generic deep brain stimulator exposed in $1.5 \mathrm{~T}$ magnetic resonance scanners," Bioelectromagnetics, vol. 34, no. 2, pp. 104-113, Feb. 2013.

[21] J. E. Brown and C. S. Lee, "Radiofrequency resonance heating near medical devices in magnetic resonance imaging," Microw. Opt. Technol. Lett., vol. 55, no. 2, pp. 299-302, Feb. 2013.

[22] "ICNIRP statement on: medical magnetic resonance (MR) procedures: protection of patients," International Commission on Non-Ionizing Radiation Protection (ICNIRP), Health Phys., vol. 87, no. 2, pp. 197216, Aug. 2004.

[23] S. McCabe and J. Scott, "Technique to assess the compatibility of medical implants to the RF field in MRI," Asia-Pacific Microwave Conference. Nanjing, 6-9 Dec. 2015, pp. 1-3.

[24] P. A. Bottomley, A. Kumar, W. A. Edelstein, J. M. Allen, and P. V. Karmarkar, "Designing passive MRI-safe implantable conducting leads with electrodes," Med. Phys., vol. 37, no. 7, pp. 3828-3843, Jun. 2010.

[25] C. Jiang, X. Mo, J. Ding, Y. Dong, F. Zhang, H. Hao, and L. Li, "Deep brain stimulation lead design to reduce radio frequency heating in MRI", Electronics Letters, vol. 50, no. 25, pp. 1898-1900, Dec. 2014.

[26] S. Ramo, J. R. Whinnery, and T. Van Duzer, Fields and waves in communication electronics, second edition, John Wiley \& Sons, Inc, pp. 147-150, 1984.

[27] R. W. P. King and G. S. Smith, Antennas in matter: fundamentals, theory, and applications, The MIT Press, Cambridge, MA, 1981

[28] F2182-11a standard test method for measurement of radio frequency induced heating on or near passive implants during magnetic resonance imaging, ASTM International, West Conshohocken, PA, USA, 2010.

[29] K. R. Gorny, M. F. Presti, S. J. Goerss, S. C. Hwang, D. Jang, I. Kim, H. Min, Y. Shu, C. P. Favazza, K. H. Lee, and M. A. Bernstein, "Measurements of RF heating during 3.0-T MRI of a pig implanted with deep brain stimulator," Magn. Reson. Imag., vol. 31, no. 5, pp. 783-788, Jun. 2013.
[30] K. B. Baker, J. A. Tkach, J. A. Nyenhuis, M. Phillips, F. G. Shellock, J. Gonzalez-Martinez, and A. R. Rezai, "Evaluation of specific absorption rate as a dosimeter of MRI-related implant heating," J. Magn. Resonance Imaging, pp. 315-320, Aug. 2004.

[31] A. R. Rezai, D. Finelli, J. A. Nyenhuis, G. Hrdlicka, J. A. Tkach, A. Sharan, P. Rugieri, P. H. Stypulkowski, and F. G. Shellock, "Neurostimulation systems for deep brain stimulation: in vitro evaluation of magnetic resonance imaging-related heating at 1.5 Tesla," J. Magn. Resonance Imaging, pp. 241-250, 2002.

[32] Assessment of the safety of magnetic resonance imaging for patients with an active implantable medical device, Technical specification ISO/TS 10974, 2012.

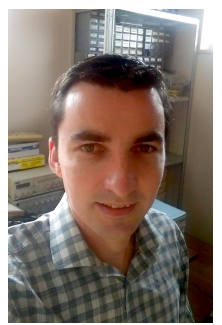

Steven McCabe received a B.E in electronic engineering and an M.Phil. in microwave engineering at the University of Waikato, New Zealand, in 2009 and 2011, respectively. He is working toward the Ph.D. degree in electronic engineering at the University of Waikato.

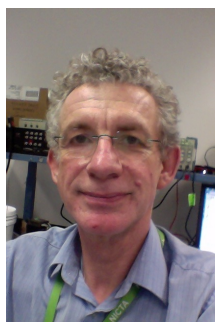

Jonathan Scott (M'80-SM'99) is the Foundation Professor in Electronic Engineering at the University of Waikato, New Zealand. From 1998 to 2006 he was with the Hewlett-Packard and Agilent Technologies Microwave Technology Center in Santa Rosa, California, where he was responsible for advanced measurement systems. In 1997 and 1998 he was Chief Engineer at RF Technology in Sydney. He was with the University of Sydney in the Department of Electrical Engineering prior to 1997. Professor Scott holds five degrees, has authored over 100 refereed publications, several book chapters and a textbook, and he holds a dozen patents, several covering active products. Professor Scott's educational interests include Threshold Concepts and their application, particularly across engineering disciplines. 